\title{
Inflammation and Coronary Heart Disease Risk in Patients with Depression in China Mainland: A Cross-Sectional Study
}

This article was published in the following Dove Press journal: Neuropsychiatric Disease and Treatment

\section{Lina Zhou \\ Xiancang $\mathrm{Ma}$ (iD \\ Wei Wang}

Department of Psychiatry, The First Affiliated Hospital of Xi'an Jiaotong University, Xi'an, Shaanxi, People's Republic of China
Correspondence: Wei Wang Department of Psychiatry, The First Affiliated Hospital of Xi'an Jiaotong University, 277 West Yanta Road, Xi'an, Shaanxi, People's Republic of China Tel +8602985323II2

Email xianwv@sina.com
Objective: The risk of coronary heart disease (CHD) in patients with major depressive disorder (MDD) is higher than that in the general population. However, the mechanisms underlying the increased CHD risk in patients with MDD remain unclear. Inflammation plays an important role in the pathogenesis of MDD and CHD. Therefore, we explored the relationship between inflammatory biomarkers and CHD risk in patients with MDD

Methods: We included 454 patients with acute MDD and 458 controls that matched the sample in age and gender. A readily available complete blood count was used to reflect inflammation, and the risk of CHD was assessed using the Framingham risk score.

Results: The results showed that patients with MDD showed low-grade inflammation with an elevated platelet $(\mathrm{p}<0.001)$ and monocyte count $(\mathrm{p}<0.001)$, high platelet/lymphocyte $(\mathrm{p}=0.003)$ and monocyte/lymphocyte ratios $(\mathrm{p}<0.001)$, and a raised systemic immuneinflammation index $(\mathrm{p}=0.002)$. In addition, monocyte count was the only factor significantly associated with CHD risk in patients with MDD (B=7.521, 95\% CI: $3.409-11.633, \mathrm{t}=3.594$, $\mathrm{p}<0.001)$.

Conclusion: Collectively, the results of this study support the hypothesis that MDD is systemic inflammation, and suggest that monocyte count predicts the risk of CHD in patients with MDD.

Keywords: major depressive disorder, inflammation, coronary heart disease, complete blood count, Framingham risk score

\section{Introduction}

Major depressive disorder (MDD) is a kind of mental illness with a lifetime prevalence of $16.2 \%$, and around 300 million people worldwide suffer from MDD. ${ }^{22}$ Especially in China, the prevalence of MDD increases year by year and leads to high medical and social burden. ${ }^{9}$ Patients with MDD experience depressed mood, loss of interest and enjoyment, and reduced energy, which leads to diminished activity. At its worst, MDD can lead to suicide. ${ }^{4}$ Close to 800,000 people die owing to suicide every year, and suicide has become the second leading cause of death in $15-29$-year-olds. ${ }^{19}$ It is apparent that we cannot avoid the challenge of MDD.

To amplify these grim facts, the pathogenesis of MDD is still unclear. Recently, inflammation has been connected to the etiopathogenesis of MDD by regulating hypothalamic-pituitary-adrenal (HPA) axis activation, neurotransmitter systems, and neuroplasticity, which has been provided evidence with cytokines. ${ }^{8}$ Some indicators of complete blood count (CBC) are simple and sensitive to reflect inflammatory changes 
in the body, such as the count of white blood cells (WBC), monocytes, neutrophils, and lymphocytes, and neutrophil/ lymphocyte ratio (NLR), platelet/lymphocyte ratio (PLR), monocyte/lymphocyte ratio (MLR), and systemic immuneinflammation index (SIII). For example, Shafiee et al, ${ }^{18}$ found that higher depression scores were associated with an enhanced inflammatory state, which was characterized by elevated WBC. Further, Euteneuer et al, ${ }^{6}$ suggested that monocyte and neutrophil count of patients with MDD were higher than that of the control group. These results provided evidence that inflammation, as represented by a CBC, may play an important role in the pathogenesis of MDD.

People with MDD have a higher risk of coronary heart disease (CHD) than the general population. ${ }^{11,15,25}$ MDD has been considered an independent risk factor in the development of $\mathrm{CHD}^{3}$ as well as a predictor of poor prognosis of cardiovascular events. ${ }^{7}$ Therefore, exploring the mechanism of the increased risk of CHD in patients with MDD is of great importance for the early identification of and intervention for CHD in patients with MDD. Several factors seem to link MDD with cardiovascular events, including poor adherence to treatment, sympathetic stimulation, endothelial dysfunction, low heart rate variability, inflammation, and abnormal platelet function. ${ }^{2}$ Moreover, inflammation has been identified by some studies as a risk predictor of CHD. For example, procalcitonin (PCT), hypersensitive C-reactive protein (hsCRP), ${ }^{13}$ and $\mathrm{MLR}^{17}$ have been used as indicators of CHD risk. However, there are few studies focused on the role of inflammation in predicting the risk of CHD in patients with MDD. Based on this information, this study used the Framingham risk score (FRS) to evaluate the risk of CHD in patients with MDD; and the whole blood cell count, as simple and readily available indicators, were used to reflect the inflammation, so as to explore the relationship between inflammation in patients with MDD and the risk of CHD. We had three preliminary hypotheses: (1) the risk of CHD in patients with MDD will show greater increase compared to normal controls; (2) inflammatory markers in patients with MDD will be higher than those in normal controls; and (3) inflammatory indicators may increase the risk of CHD in patients with MDD.

\section{Methods}

\section{Participants}

All patient subjects were inpatients, who were recruited from the Department of Psychiatry of the First Affiliated Hospital of Xi'an Jiaotong University. The inclusion criteria of patients were as follows. The patients: (1) were diagnosed with MDD according to the fourth edition of the American Diagnostic and Statistical Manual of Mental Disorders (DSM-IV) within a week of selection; (2) were aged between 30 and 74 years; (3) were without severe somatic diseases, especially cardiovascular and cerebrovascular diseases, and any other diseases which might affect endocrine, metabolic, hepatic, or renal functioning; (4) currently had no other diseases that meet the diagnostic criteria of DSM-IV; and (5) had no previous history of CHD and stroke. Healthy controls were recruited from the Physical Examination Center of the First Affiliated Hospital of Xi'an Jiaotong University, and their inclusion criteria were as follows. The control subjects: (1) did not meet any diagnostic criteria of DSM-IV; (2) were aged between 30 and 74 years; (3) were without severe somatic diseases, especially cardiovascular and cerebrovascular diseases, and diseases which might affect endocrine, metabolic, hepatic, or renal functioning; and (4) had no previous history of CHD and stroke.

All participants received a detailed introduction about the study, following which they gave their written, informed consent. Following the ethical standards for human research, this study was conducted in accordance with the declaration of Helsinki and approved by the Ethics Committee of the First Affiliated Hospital of Xi'an Jiaotong University (Ethical approval: No. XJTU1AF2018LSK-076).

\section{Complete Blood Count}

Blood samples were obtained between 6:00 and 9:00 a.m. on the day following enrollment after an overnight fast. The overnight fasting was supervised. Immediately after collecting blood samples, CBC was determined using Sysmex XS-1000i TM Automated Hematology Analyzer (Sysmex, USA). Absolute numbers of WBC, neutrophils, monocytes, lymphocytes, and platelets were measured. NLR, PLR, and MLR are calculated as the neutrophil/ lymphocyte ratio, the platelet/lymphocyte ratio, and monocyte/lymphocyte ratio, respectively. SIII is conducted as $\mathrm{A} * \mathrm{~B} / \mathrm{C}$, where A represents platelet count, $\mathrm{B}$ represents neutrophil count, and $\mathrm{C}$ represents lymphocyte count.

\section{0-Year Framingham CHD Risk Assessment}

FRS is a scoring system that uses age, gender, smoking, blood pressure, total cholesterol, high-density lipoprotein (HDL), and diabetes to estimate coronary event risk over 
the course of 10 years among individuals with no previously diagnosed CHD. ${ }^{23,24}$ In this study, we evaluated the CHD risk of subjects based on the data available in the Framingham Heart Study web page (https://www.framinghamheartstudy. org/fhs-risk-functions/coronary-heart-disease-10-year-risk).

\section{Data Analysis}

SPSS 24.0 (SPSS Inc., Chicago, Illinois, USA) was used for statistical analysis. Categorical variables were analyzed by the Chi-square test. Continuous variables (mean \pm standard deviation) were first tested for normalcy using the Kolmogorov-Smirnov test. Normally distributed parameters were further tested using a two-tailed independent sample $t$-test, and non-normal distribution parameters were analyzed using a non-parametric test, namely the MannWhitney $U$-test. The correlations of FRS and factors were analyzed by Pearson correlation analysis and stepwise linear regression analysis. Non-standardized coefficients (B values) with $95 \%$ confidence intervals $(95 \% \mathrm{CI})$ were computed. All statistical tests were two-tailed, and differences were considered significant at $\mathrm{p}<0.05$.

\section{Results}

The demographic and clinical information of all the subjects are listed in Table 1. The participants included 458 healthy subjects and 454 patients with MDD, who were matched for age $(\mathrm{t}=-0.116, \mathrm{p}=0.908)$ and gender $\left(\chi^{2}=1.465, \mathrm{p}=0.226\right)$. The average FRS among healthy control group subjects was $2.36 \pm 1.13 \%$ and $6.46 \pm 4.93 \%$ for patients with MDD. For patients with MDD, their average illness duration and medication duration were $72.29 \pm 84.66$ months and $52.94 \pm 70.10$ months, respectively. Compared to the healthy control group, the MDD group had more subjects with smoking, hypertension, and diabetes, lower serum high-density lipoprotein levels, and higher SBP, DBP, and FRS $(\mathrm{p}<0.05)$. Additionally, there was no significant difference in total cholesterol levels between healthy controls and patients with MDD ( $p>0.05)$.

The CBC of all subjects is listed in Table 2. Compared to healthy controls, patients with MDD showed higher platelet and monocyte count, PLR, MLR, and SIII $(\mathrm{p}<0.05)$.

To explore the relationship between $\mathrm{CBC}$ and FRS, we conducted Pearson correlation analysis and linear regression analysis. Pearson correlation analysis showed that FRS was positively correlated with monocyte count among patients with MDD ( $r=0.159, \mathrm{p}=0.001$; See Figure 1). All inflammatory markers and demographic and clinical data were included in the linear regression analysis. We found that
Table I The Demographic and Clinical Information of Healthy Controls and Patients with Depression

\begin{tabular}{|c|c|c|c|c|}
\hline & HC $(n=458)$ & $M D(n=454)$ & Statistic & $\mathbf{p}$ \\
\hline \multicolumn{5}{|l|}{ Gender } \\
\hline Male, n (\%) & 147 & 129 & 1.465 & 0.226 \\
\hline Female, $n(\%)$ & 311 & 325 & & \\
\hline Age, year, $M \pm S D$ & $53.02 \pm 6.75$ & $53.08 \pm 9.20$ & -0.116 & 0.908 \\
\hline \multicolumn{5}{|l|}{ Smoking } \\
\hline Yes, n (\%) & 8 & 36 & $|8.98|$ & $<0.001$ \\
\hline No, n (\%) & 450 & 418 & & \\
\hline $\mathrm{SBP}, \mathrm{mmHg}, \mathrm{M} \pm \mathrm{SD}$ & $111.48 \pm 14.18$ & $121.02 \pm 16.03$ & -9.516 & $<0.001$ \\
\hline $\mathrm{DBP}, \mathrm{mmHg}, \mathrm{M} \pm \mathrm{SD}$ & $74.5 I \pm 8.27$ & $78.26 \pm 9.68$ & -6.289 & $<0.001$ \\
\hline \multicolumn{5}{|l|}{ Hypertension } \\
\hline Yes, n (\%) & 45 & 95 & 20.359 & $<0.001$ \\
\hline No, n (\%) & 403 & 359 & & \\
\hline \multicolumn{5}{|l|}{ Diabetes } \\
\hline Yes, n (\%) & 3 & 28 & 21.099 & $<0.001$ \\
\hline No, n (\%) & 455 & 426 & & \\
\hline $\mathrm{TC}, \mathrm{mmol} / \mathrm{L}, \mathrm{M} \pm \mathrm{SD}$ & $4.25 \pm 0.69$ & $4.29 \pm 0.90$ & -0.206 & 0.837 \\
\hline $\begin{array}{l}\mathrm{HDL}, \mathrm{mmol} / \mathrm{L}, \mathrm{M} \\
\pm \mathrm{SD}\end{array}$ & $1.36 \pm 0.27$ & $1.15 \pm 0.27$ & -10.992 & $<0.001$ \\
\hline $\begin{array}{l}\text { Illness duration, } \\
\text { month, } \mathrm{M} \pm \mathrm{SD}\end{array}$ & & $72.29 \pm 84.66$ & - & - \\
\hline $\begin{array}{l}\text { Medication } \\
\text { duration, month, M } \\
\pm S \mathrm{SD}\end{array}$ & & $52.94 \pm 70.10$ & - & - \\
\hline $\begin{array}{l}\text { Attack times, } M \\
\pm S D\end{array}$ & & $2.02 \pm 1.44$ & - & - \\
\hline Family history & & & & \\
\hline Yes, n (\%) & & 60 & - & - \\
\hline No, n (\%) & & 394 & & \\
\hline FRS, \%, M $\pm S D$ & $2.36 \pm 1.13$ & $6.46 \pm 4.93$ & -17.680 & $<0.001$ \\
\hline
\end{tabular}

Notes: Bold data indicates statistical significance.

Abbreviations: $\mathrm{HC}$, healthy control; MD, patients with major depression; SBP, systolic blood pressure; DBP, diastolic blood pressure; TC, total cholesterol; HDL, high-density lipoprotein; FRS, Framingham risk score; M $\pm S D$, mean \pm standard deviation.

monocyte count ( $\mathrm{B}=7.521,95 \% \mathrm{CI}: 3.409-11.633$, $\mathrm{t}=3.594$, $\mathrm{p}<0.001$ ) was significantly correlated with FRS in patients with MDD, while this finding was not found in healthy controls $(\mathrm{B}=-1.457,95 \% \quad \mathrm{CI}:-6.012-3.097, \mathrm{t}=-0.629$, $\mathrm{p}=0.530)$.

\section{Discussion}

Inflammation is considered to be one of the underlying mechanisms leading to $\mathrm{MDD}{ }^{8} \mathrm{CBC}$ is a simple and sensitive indicator to evaluate the level of inflammation in 
Table 2 The Complete Blood Count of Heathy Controls and Patients with Depression

\begin{tabular}{|c|c|c|c|c|}
\hline & $\mathrm{HC}$ & MD & Statistic & $\mathbf{p}$ \\
\hline \multirow[t]{2}{*}{$\mathrm{Plt}, 10^{9} / \mathrm{L}$} & 190.60 & 208.03 & -3.693 & $<0.001$ \\
\hline & \pm 43.71 & \pm 63.28 & & \\
\hline WBC, $10^{9} / \mathrm{L}$ & $5.36 \pm 1.09$ & $5.5 I \pm 2.89$ & -0.443 & 0.657 \\
\hline $\mathrm{N}, 10^{9} / \mathrm{L}$ & $3.24 \pm 0.88$ & $3.35 \pm 1.38$ & -0.048 & 0.962 \\
\hline $\mathrm{L}, 10^{9} / \mathrm{L}$ & $|.7| \pm 0.43$ & $1.75 \pm 0.56$ & -0.228 & 0.820 \\
\hline $\mathrm{M}, 10^{9} / \mathrm{L}$ & $0.27 \pm 0.08$ & $0.30 \pm 0.11$ & $-4.74 I$ & $<0.001$ \\
\hline NLR & $2.00 \pm 0.73$ & $2.09 \pm 1.45$ & -0.683 & 0.495 \\
\hline \multirow[t]{2}{*}{ PLR } & 117.20 & 127.86 & -2.975 & 0.003 \\
\hline & \pm 36.97 & \pm 52.45 & & \\
\hline MLR & $0.16 \pm 0.05$ & $0.18 \pm 0.08$ & -3.699 & $<0.001$ \\
\hline \multirow[t]{2}{*}{ SIII } & 379.52 & 437.56 & -3.114 & 0.002 \\
\hline & \pm 164.74 & \pm 361.73 & & \\
\hline
\end{tabular}

Notes: Bold data indicates statistical significance.

Abbreviations: $\mathrm{HC}$, healthy control; MD, patients with major depression; Plt, platelet, WBC, white blood cell; $N$, neutrophil; L, lymphocyte; $M$, monocyte; NLR, neutrophil/lymphocyte radio; PLR, platelet/lymphocyte radio; MLR, monocyte/lymphocyte radio; SIII, systemic immune-inflammation index.

patients with MDD. A total of 458 healthy controls and 454 MDD patients (both groups matching in gender and age) were included in this study, and we found that the inflammatory markers, including platelet and monocyte count, PLR, MLR, and SIII, in the whole blood of MDD patients were significantly higher than those in the normal control group. These results suggested that patients with MDD had more active inflammation. Particularly, SIII, as an indicator of systemic immune inflammation, was found to be significantly higher in patients with MDD than in the control group, suggesting that MDD may be a product of systemic chronic inflammation. In addition, we also used the FRS to evaluate the 10-year CHD risk of patients with

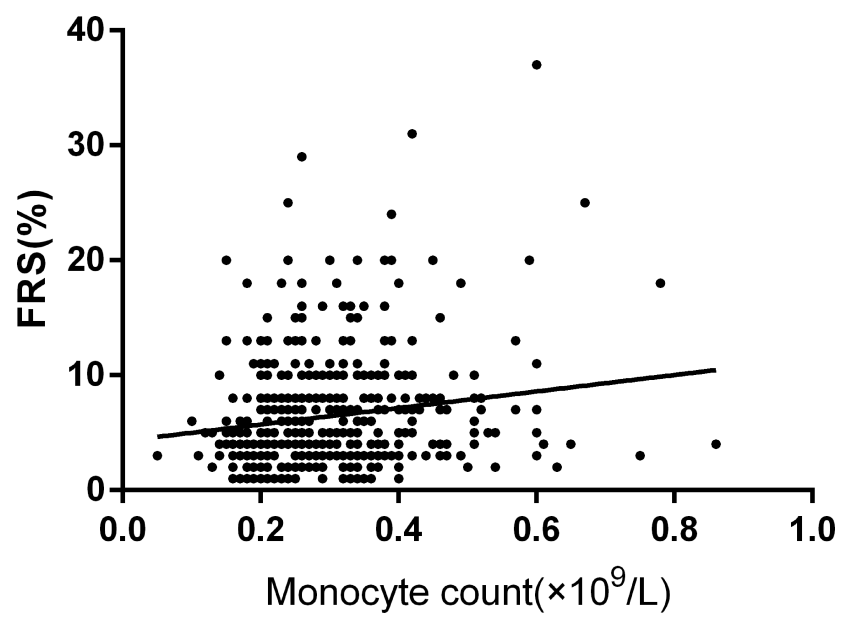

Figure I Correlations between FRS and monocyte count.
MDD and found that the 10-year CHD risk of patients with MDD was significantly higher than that of healthy controls, whose scores were $6.46 \%$ and $2.36 \%$, respectively. Considering that inflammation plays an important role both in MDD and CHD, we explored the relationship between them, and the results showed that monocyte count of patients with MDD was the only factor that significantly correlated with their CHD risk, which showed that inflammation plays an important role in increased CHD risk in patients with MDD on the one hand, and monocyte count might be used to predict the risk of CHD on the other hand.

Some studies have explored the relationship between the risk of CHD and depressive symptoms using the FRS. For example, Koponen et $\mathrm{al}^{12}$ concluded that more severe MDD is related to a higher risk of CHD. However, these studies were conducted among the general population using self-rated or other-rated scales to assess depressive symptoms. Few studies have assessed the risk of CHD in patients with diagnosed MDD. Therefore, in this study, we took the hospitalized patients with MDD as the participants of the study and evaluated the risk of CHD in the acute phase, which can reflect the risk of CHD in patients with MDD more effectively.

Parissis et al $^{16}$ concluded that MDD and CHD share a number of common underlying mechanisms, including hypercoagulability via platelet activation, hypothalamuspituitary-adrenal axis and autonomic nervous system dysregulation, and altered inflammatory response. In particular, in this study, we compared a few inflammatory indicators in MDD patients and members of the control group, and found that patients with MDD had higher counts of monocytes and platelets, MLR, PLR and SIII. These results suggested that MDD patients had a higher inflammatory level, and the inflammation in patients with MDD may be systemic. This coincides with some hypotheses put forward in recent years. In other words, some researchers believe that MDD or mental illnesses are not only functional disorders of the brain, but systemic changes of multiple organs that affect inflammation, metabolism, endocrine function, and so on. ${ }^{5,20}$ Berk et $\mathrm{al}^{1}$ concluded that psychosocial stressors, poor diet, physical inactivity, obesity, smoking, altered gut permeability, atopy, dental issues, sleep problems, and vitamin D deficiency seem to be associated with systemic inflammation in patients with MDD.

As an important component of the defense system, monocytes participate in the immune response and deliver 
the epitope to lymphocytes after phagocytosis to induce the specific immune response of lymphocytes. Macrophages (mostly derived from monocytes) have a fundamental role in the formation and progression of coronary atherosclerotic plaques; and Waterhouse et al, ${ }^{21}$ suggested the monocyte count has the strongest positive and independent relationship with cardiovascular disease (CVD) risk in asymptomatic adults. Moreover, monocyte count can predict the severity and progression of atherosclerotic stenosis in acute coronary syndrome. ${ }^{10,14}$ Therefore, these results provide evidence for the role of monocyte count in predicting CVD risk. In this study, we explored the relationship between inflammation and CHD risk in patients with MDD, and we found that monocyte count was the only significant indicator that correlated with their CHD risk, both analyzed by Pearson correlation and linear regression analysis. It revealed that activated inflammation may contribute to an increased risk of CHD in people with MDD. This is the first demonstration of the importance of monocyte count in predicting the risk of CHD in patients with MDD. To our knowledge, this has never been reported before.

This study has three limitations. First, studies have reported that the severity of depressive symptoms is related to the risk of CHD. ${ }^{25}$ In this study, we did not assess the severity of MDD using scales, although we have ensured that all patients were in the acute phase. Second, we stipulated strict inclusion criteria for healthy controls, especially for items that related to the FRS, such as hyperlipidemia; this may have led to a lower risk of CHD in the healthy controls. Third, as we did not follow up with patients with MDD after the study, it was not checked whether inflammation is a trait or state marker; this should be explored further in future.

In conclusion, we found that patients with MDD show a low-grade inflammation in this study, which could lead to an elevated risk of CHD. In addition, we found that monocyte count predicts the risk of CHD in patients with MDD. This provides a basis for early clinical evaluation and reduction of CHD risk in patients with MDD.

\section{Acknowledgment}

The authors thank all the participants for their support in this study.

\section{Funding}

This study was supported by the National Natural Science Foundation of China (no. 81771471) and Shaanxi
Provincial Key Research and Development Program (no.2017ZDXM-SF-25-6) and Shaanxi Province Innovation Capacity Support Program (no.2017KCT-20).

\section{Disclosure}

All authors have no conflict of interest.

\section{References}

1. Berk M, Williams LJ, Jacka FN, O’Neil A, Pasco JA, Moylan S. So depression is an inflammatory disease, but where does the inflammation come from? BMC Med. 2013;11:200. doi:10.1186/1741-7015$11-200$

2. Bounhoure JP, Galinier M, Curnier D, Bousquet M, Bes A. [Influence of depression on the prognosis of cardiovascular diseases (in French)]. Bull Acad Natl Med. 2006;190(8):1723-1731.

3. Charlson FJ, Stapelberg NJ, Baxter AJ, Whiteford HA. Should global burden of disease estimates include depression as a risk factor for coronary heart disease? BMC Med. 2011;9:47. doi:10.1186/17417015-9-47

4. Cho SE, Na KS, Cho SJ, Im JS, Kang SG. Geographical and temporal variations in the prevalence of mental disorders in suicide: systematic review and meta-analysis. $J$ Affect Disord. 2016;190:704-713. doi:10.1016/j.jad.2015.11.008

5. Dantzer R, O'Connor JC, Freund GG, Johnson RW, Kelley KW. From inflammation to sickness and depression: when the immune system subjugates the brain. Nat Rev Neurosci. 2008;9(1):46-56. doi:10.1038/nrn2297

6. Euteneuer F, Dannehl K, Del Rey A, Engler H, Schedlowski M, Rief W. Immunological effects of behavioral activation with exercise in major depression: an exploratory randomized controlled trial. Transl Psychiatry. 2017;7(5):e1132. doi:10.1038/tp.2017.76

7. Fioranelli M, Bottaccioli AG, Bottaccioli F, Bianchi M, Rovesti M, Roccia MG. Stress and inflammation in coronary artery disease: a review psychoneuroendocrineimmunology-based. Front Immunol. 2018;9:2031. doi:10.3389/fimmu.2018.02031

8. Han QQ, Yu J. Inflammation: a mechanism of depression? Neurosci Bull. 2014;30(3):515-523. doi:10.1007/s12264-013-1439-3

9. Hsieh CR, Qin X. Depression hurts, depression costs: the medical spending attributable to depression and depressive symptoms in China. Health Econ. 2018;27(3):525-544. doi:10.1002/hec.3604

10. Huang G, Zhong XN, Zhong B, et al. Significance of white blood cell count and its subtypes in patients with acute coronary syndrome. Eur J Clin Invest. 2009;39(5):348-358. doi:10.1111/j.1365-2362.2009. 02107.x

11. Jang HY, Song YK, Kim JH, et al. Impact of depression on change in coronary heart disease risk status: the Korean Genome and Epidemiology Study (KoGES). Ther Clin Risk Manag. 2018;14:121-128. doi:10.2147/TCRM.S149501

12. Koponen H, Jokelainen J, Keinänen-Kiukaanniemi S, Vanhala M. Depressive symptoms and 10-year risk for cardiovascular morbidity and mortality. World J Biol Psychiatry. 2010;11(6):834-839. doi:10.3109/15622975.2010.486842

13. Kunutsor SK, Flores-Guerrero JL, Kieneker LM, et al. Plasma calprotectin and risk of cardiovascular disease: findings from the PREVEND prospective cohort study. Atherosclerosis. 2018;275:205-213. doi:10.1016/j.atherosclerosis.2018.06.817

14. Nozawa N, Hibi K, Endo M, et al. Association between circulating monocytes and coronary plaque progression in patients with acute myocardial infarction. Circ J. 2010;74(7):1384-1391. doi:10.1253/ circj.CJ-09-0779

15. O'Neil A, Fisher AJ, Kibbey KJ, et al. The addition of depression to the Framingham risk equation model for predicting coronary heart disease risk in women. Prev Med. 2016;87:115-120. 
16. Parissis JT, Fountoulaki K, Filippatos G, Adamopoulos S, Paraskevaidis I, Kremastinos D. Depression in coronary artery disease: novel pathophysiologic mechanisms and therapeutic implications. Int J Cardiol. 2007;116(2):153-160. doi:10.1016/j.ijcard.2006.03.038

17. Serföző G, Horváth T, Földesi I, Rafael B, von Känel R, Keresztes M. The monocyte-to-lymphocyte ratio correlates with psycho-neuro-inflammatory factors in patients with stable coronary artery disease. Neuroimmunomodulation. 2016;23(2):67-74. doi:10. $1159 / 000443835$

18. Shafiee M, Tayefi M, Hassanian SM, et al. Depression and anxiety symptoms are associated with white blood cell count and red cell distribution width: a sex-stratified analysis in a population based study. Psychoneuroendocrinology. 2017;84:101-108. doi:10.1016/j. psyneuen.2017.06.021

19. Sun XJ, Niu GF, You ZQ, Zhou ZK, Tang Y. Gender, negative life events and coping on different stages of depression severity: a cross-sectional study among Chinese University students. J Affect Disord. 2017;209:177-181.
20. Varan Ö, Babaoğlu H, Göker B. Associations between depressive disorders and inflammatory rheumatic diseases. Curr Top Med Chem. 2018;18(16):1395-1401. doi:10.2174/1568026618666180516100805

21. Waterhouse DF, Cahill RA, Sheehan F, McCreery C. Prediction of calculated future cardiovascular disease by monocyte count in an asymptomatic population. Vasc Health Risk Manag. 2008;4 (1):177-187. doi:10.2147/VHRM.S2240

22. WHO. 2012. Available from: https://www.who.int/zh/news-room /fact-sheets/detail/depression.

23. Wilson PWF, D’Agostino RB, Levy D, Belanger AM, Silbershatz H, Kannel WB. Prediction of Coronary heart disease using risk factor categories. Circulation. 1998a;97:1837-1847. doi:10.1161/01.CIR. 97.18.1837

24. Wilson PWF. Estimating risk of CHD in women. Circulation. 1998b;97:1837-1847.

25. Yakar B, Ertekin YH. Screening cardiovascular risk in patients with high depression scores. Pak J Med Sci. 2018;34(3):610-615. doi:10.12669/pjms.343.14560

\section{Publish your work in this journal}

Neuropsychiatric Disease and Treatment is an international, peerreviewed journal of clinical therapeutics and pharmacology focusing on concise rapid reporting of clinical or pre-clinical studies on a range of neuropsychiatric and neurological disorders. This journal is indexed on PubMed Central, the 'PsycINFO' database and CAS, and is the official journal of The International Neuropsychiatric Association (INA). The manuscript management system is completely online and includes a very quick and fair peer-review system, which is all easy to use. Visit http://www.dovepress.com/testimonials.php to read real quotes from published authors. 